\title{
Existence of time periodic solutions for a class of non-resonant discrete wave equations
}

\author{
Guang Zhang ${ }^{1 *}$, Wenying Feng ${ }^{2}$ and Yubin Yan ${ }^{3}$
}

\section{"Correspondence:}

Ixyzhg@tjcu.edu.cn

${ }^{1}$ School of Science, Tianjin

University of Commerce, Tianjin,

300134, P.R. China

Full list of author information is

available at the end of the article

\begin{abstract}
In this paper, a class of discrete wave equations with Dirichlet boundary conditions are obtained by using the center-difference method. For any positive integers $m$ and $T$, when the existence of time $m T$-periodic solutions is considered, a strongly indefinite discrete system needs to be established. By using a variant generalized weak linking theorem, a non-resonant superlinear (or superquadratic) result is obtained and the Ambrosetti-Rabinowitz condition is improved. Such a method cannot be used for the corresponding continuous wave equations or the continuous Hamiltonian systems; however, it is valid for some general discrete Hamiltonian systems.
\end{abstract}

Keywords: wave equation; Hamiltonian system; strongly indefinite discrete system; time $m T$-periodic solution; variant generalized weak linking theorem; Ambrosetti-Rabinowitz condition

\section{Introduction}

The existence of time periodic solutions for a nonlinear wave equation of the form

$$
u_{t t}-u_{x x}+g(t, u)=0, \quad 0<x<\pi, t \in R,
$$

with the Dirichlet boundary conditions

$$
u(0, t)=0=u(\pi, t)
$$

had been established in Vitt [1] when the distributed self-oscillating systems been considered.

We note that the eigenvalues of the operator $\partial_{t t}-\partial_{x x}$ in the space of functions $u(x, t)$, $2 \pi / \omega$-periodic in time and such that, say, $u(\cdot, t) \in H_{0}^{1}(0, \pi)$ for all $t$, are $-\omega^{2} l^{2}+j^{2}, l \in Z$, $j \geq 1$. Therefore, when $\omega^{2}$ is irrational, the eigenvalues accumulate to 0 . In this case, the inverse operator of $\partial_{t t}-\partial_{x x}$ is unbounded and the standard implicit function theorem is not applicable. When $\omega^{2}$ is rational, the number of 0 -spectra is infinite, thus, this will introduce the presence of an infinite-dimensional bifurcation equation. Consequently, when we consider the existence of the time periodic solutions for problem (1)-(2), two main difficulties must be overcome: the 'small denominators' problem and the presence of an 
infinite-dimensional bifurcation equation. To this end, two main methods are used, that is, the variations viewpoint (see Rabinowitz [2] and Brézis and Coron [3]) and the KAM theory (see Berti and Bolle [4] and Gentile and Mastropietro [5]).

Let $Z$ be a set of all integers, $R$ be a set of all real numbers, and $Z^{+}=\{0,1,2, \ldots\}$. For any integers $k$ and $l$ with $k<l$, denote $[k, l]=\{k, k+1, \ldots, l\}$. By using the center-difference method for the space variable $x$ and the time variable $t$, we can obtain a discrete analog of (1)-(2) of the form

$$
\left\{\begin{array}{l}
\frac{1}{h^{2}} \Delta^{2} u\left(\frac{i \pi}{N+1},(n-1) h\right)-\left(\frac{N+1}{\pi}\right)^{2} \nabla^{2} u\left(\frac{(i-1) \pi}{N+1}, n h\right) \\
\quad+g\left(n h, u\left(\frac{i \pi}{N+1}, n h\right)\right)=0, \quad i \in[1, N], n \in Z \\
u\left(\frac{0 \pi}{N+1}, n h\right)=u\left(\frac{N+1) \pi}{N+1}, n h\right)=0, \quad n \in Z
\end{array}\right.
$$

where $h>0$ is the time step size, $N$ is a positive integer and the space step size is $\pi /(N+1)$.

Let

$$
u\left(\frac{i \pi}{N+1}, n h\right)=u_{n}^{i}
$$

then we have

$$
\left\{\begin{array}{l}
\Delta^{2} u_{n-1}^{i}-\delta^{2} \nabla^{2} u_{n}^{i-1}+f\left(n, u_{n}^{i}\right)=0, \quad i \in[1, N], n \in Z, \\
u_{n}^{0}=0=u_{n}^{N+1}, \quad n \in Z,
\end{array}\right.
$$

where

$$
\begin{aligned}
& \Delta^{2} u_{n-1}^{i}=u_{n+1}^{i}-2 u_{n}^{i}+u_{n-1}^{i}, \\
& \nabla^{2} u_{n}^{i-1}=u_{n}^{i+1}-2 u_{n}^{i}+u_{n}^{i-1}, \\
& \delta^{2}=h^{2}\left(\frac{N+1}{\pi}\right)^{2},
\end{aligned}
$$

and

$$
f\left(n, u_{n}^{i}\right)=h^{2} g\left(n h, u\left(\frac{i \pi}{N+1}, n h\right)\right) .
$$

Problem (4) can be rewritten by the vector and matrix as

$$
\Delta^{2} U_{n-1}+\delta^{2} A U_{n}+\nabla V\left(n, U_{n}\right)=0, \quad n \in Z,
$$

where

$$
\begin{aligned}
& U_{n}=\operatorname{col}\left(u_{n}^{1}, u_{n}^{2}, u_{n}^{N}\right) \\
& A=\left(\begin{array}{ccccc}
2 & -1 & 0 & \cdots & 0 \\
-1 & 2 & -1 & & 0 \\
& \ldots & & \ldots & \\
0 & & -1 & 2 & -1 \\
0 & \cdots & 0 & -1 & 2
\end{array}\right)_{N \times N},
\end{aligned}
$$


and

$$
V\left(n, U_{n}\right)=\sum_{i=1}^{N} \int_{0}^{u_{n}^{i}} f(n, s) d s
$$

which implies that

$$
\nabla V\left(n, U_{n}\right)=\operatorname{col}\left(f\left(n, u_{n}^{1}\right), f\left(n, u_{n}^{2}\right), \ldots, f\left(n, u_{n}^{N}\right)\right)
$$

For a given positive integer $T$, we assume that the function $f: Z \times R \rightarrow R$ is continuous about the two variables and satisfies the periodic condition

$$
f(n+T, \cdot)=f(n, \cdot) \quad \text { for } n \in Z
$$

Let $L=-\left(\Delta^{2}+\delta^{2} A\right)$. For any positive integers $T$ and $m, \sigma(L)$ denotes the spectrum of the linear operator $L$ in $E_{m T}$ which will be defined in the next section. We can see that $\sigma(L) \cap(0, \infty) \neq \emptyset$ and $\sigma(L) \cap(-\infty, 0) \neq \emptyset$. In this paper, we will consider the existence of $m T$-periodic solutions for problem (4) when the condition $0 \notin \sigma(L)$ holds. However, 0 is the spectrum point of the linear operator $\partial_{t t}-\partial_{x x}$ for problem (1)-(2). Thus, our method cannot be used for the corresponding continuous wave equations.

Clearly, system (5) is also a discrete second order Hamiltonian system. Recently, the existence of $T$-periodic solutions for system (5) has also been extensively studied when $A$ is symmetric matrix and $L$ is positive definite. By using critical point theory, many solvability conditions are given, such as the superquadratic condition and subquadratic condition (see Deng et al. [6] and Yan et al. [7]), the convex condition (see Jiang [8]), and the asymptotically linear condition (see Guo and Yu [9]). Our method can be extended to a general system, however, the linear operator $L$ is strongly indefinite. On the other hand, in the superquadratic case, all papers required the Ambrosetti-Rabinowitz condition, that is, there exist constants $\mu>2$ and $M>0$ such that

$$
0<\mu H(t, U) \leq(\nabla H(t, U), U), \quad|U| \geq M, t \in[0, T]
$$

where

$$
|U|=\left(\sum_{i=1}^{N}\left|u^{i}\right|^{2}\right)^{1 / 2}, \quad U \in R^{N} .
$$

In this paper, we will give a more general condition.

In fact, system (5) is also a discrete analog of the second order Hamiltonian system of the form

$$
U^{\prime \prime}(t)+B U(t)+\nabla H(t, U)=0, \quad t \in R
$$

The existence of periodic solutions of (8) have also been extensively discussed since Poincaré [10]. The importance of periodic solutions for finite-dimensional Hamiltonian system was pointed out by Poincaré in [10]. Poincaré stressed their importance formulating a conjecture. This conjecture stimulates the systematic study of periodic solutions 
by Poincaré himself, Lyapunov [11], Birkhoff and Lewis [12], Moser [13], Weinstein [14], Rabinowitz [15], Ekeland and Lasry [16], etc. In Pugh and Robinson [17] a positive answer to the conjecture was given, but only in a generic sense (namely in the $C^{2}$-category of Hamiltonian functions): the periodic orbits are dense on every compact and regular energy surface. However, for specific systems, the conjecture is still open (and far from having been proved); see [18].

Since Rabinowitz's pioneering work [15] of 1978, variational methods have been widely used in the study of existence of solutions of Hamiltonian systems; see Long [19] and Luan and Mao [20]. Recently, some authors had improved Ambrosetti-Rabinowitz condition by using the linking theorem; for example, see Schechter and Zou [21] and Chen and Ma [22]. In [21], the authors considered the existence of solutions for a Schrödinger equation and the classical Ambrosetti-Rabinowitz condition is replaced by a general superquadratic condition: there exist constants $a>0$ and $p>2$ such that

$$
|\nabla V(t, U)| \leq a\left(1+|U|^{p-1}\right) \quad \text { for } U \in R^{N}
$$

In [22], Chen and Ma established the existence of periodic solutions of (8) by using a similar method. However, the condition (9) will be improved in our result.

In the next section of the present paper, we will give some preliminary results which will be used in the proof of the main results. The exact spectrum of the linear operator $L$ in $E_{m T}$ will be given. In this case, we easily give the conditions $0 \notin \sigma(L), \sigma(L) \cap(0, \infty) \neq \emptyset$, and $\sigma(L) \cap(-\infty, 0) \neq \emptyset$. Thus, in this paper, we only consider the non-resonant strongly indefinite problem. Our approach is based on an application of a variant generalized weak linking strongly indefinite problem developed by Schechter and Zou [21]; also see Chen and $\mathrm{Ma}$ [22]. Thus, a variant generalized weak linking theorem is also given in this section. In Section 3, our main result will be obtained by using the variant generalized weak linking theorem, and the Ambrosetti-Rabinowitz condition will be improved.

\section{Some preliminary results}

In this section, we recall some basic facts which will be used in the proof of the main results.

Let

$$
X=\left\{U=\left\{U_{n}\right\}_{n \in Z}: U_{n} \in R^{N}, n \in Z\right\} .
$$

For any given positive integers $T$ and $m, E_{m T}$ is defined by

$$
E_{m T}=\left\{U \in X: U_{n+m T}=U_{n}, n \in Z\right\} .
$$

$E_{m T}$ can be equipped with the inner product $\langle\cdot, \cdot\rangle_{m T}$ and norm $\|\cdot\|_{m T}$ as follows:

$$
\begin{aligned}
& \langle U, V\rangle_{m T}=\sum_{n=1}^{m T}\left(U_{n}, V_{n}\right)=\sum_{n=1}^{m T} \sum_{i=1}^{N} u_{n}^{i} v_{n}^{i}, \quad U, V \in E_{m T}, \\
& \|U\|_{m T}=\left(\sum_{n=1}^{m T} \sum_{i=1}^{N}\left(u_{n}^{i}\right)^{2}\right)^{1 / 2} .
\end{aligned}
$$


Clearly, we can also define one other norm for $E_{m T}$. Note that the space $E_{m T}$ is finite dimensional, thus, they are equivalent.

It is easy to see that $\left(E_{m T},\langle\cdot, \cdot\rangle_{m T}\right)$ is a finite-dimensional Hilbert space and linearly homeomorphic to $R^{m T \times N}$. For convenience, we identify $U \in E_{m T}$ with $U=\operatorname{col}\left(U_{1}, U_{2}, \ldots, U_{m T}\right)$. In this case, we will consider the existence of solutions for the discrete wave equation

$$
\Delta^{2} u_{n-1}^{i}-\delta^{2} \nabla^{2} u_{n}^{i-1}+f\left(n, u_{n}^{i}\right)=0, \quad(i, n) \in[1, N] \times[1, m T],
$$

with the space Dirichlet boundary conditions

$$
u_{n}^{0}=0=u_{n}^{N+1}, \quad n \in[1, m T]
$$

and the time periodic boundary conditions

$$
u_{0}^{i}=u_{m T}^{i} \quad \text { and } \quad u_{1}^{i}=u_{m T+1}^{i}, \quad i \in[1, N]
$$

or the discrete Hamiltonian system

$$
\Delta^{2} U_{n-1}+\delta^{2} A U_{n}+\nabla V\left(n, U_{n}\right)=0, \quad n \in[1, m T],
$$

with the time periodic boundary conditions

$$
U_{0}=U_{m T} \quad \text { and } \quad U_{1}=U_{m T+1} .
$$

Define the functional $H$ on $E_{m T}$ as follows:

$$
\begin{aligned}
H(U) & =\sum_{j=1}^{m T} \sum_{i=1}^{N}\left[\frac{1}{2}\left|u_{j+1}^{i}-u_{j}^{i}\right|^{2}-\frac{\delta^{2}}{2}\left|u_{j}^{i+1}-u_{j}^{i}\right|^{2}-\int_{0}^{u_{j}^{i}} f(j, s) d s\right] \\
& =\frac{1}{2} \sum_{j=1}^{m T} \sum_{i=1}^{N}\left(\left|\Delta u_{j}^{i}\right|^{2}-\frac{\delta^{2}}{2}\left|\nabla u_{j}^{i}\right|^{2}\right)-\sum_{j=1}^{m T} \sum_{i=1}^{N} \int_{0}^{u_{j}^{i}} f(j, s) d s \\
& =\frac{1}{2} \sum_{j=1}^{m T}\left(\Delta U_{j}, \Delta U_{j}\right)-\frac{\delta^{2}}{2} \sum_{j=1}^{m T}\left(A U_{j}, U_{j}\right)-\sum_{j=1}^{m T} H\left(j, U_{j}\right) .
\end{aligned}
$$

A vector $W \in E_{m T}$ is called a critical point of the functional $H$ if the gradient of $H$ at $W$ is zero, i.e.,

$$
\left.\frac{\partial H(U)}{u_{j}^{i}}\right|_{U=W}=0 \quad \text { for } i \in[1, N], j \in[1, m T]
$$

At the same time, $c=H(W)$ is called a critical value of $H$. So we can obtain the following result.

Lemma 1 A vector $W \in E_{m T}$ is a critical point of the functional $H(U)($ or $-H(U))$ if, and only if, $W$ is a solution of problem (10)-(12), in fact, it is also a solution of (13)-(14). 
Its proof is similar to Lemma 1 in [23], thus, it will be omitted.

In the following, we will consider the eigenvalue problem of the form

$$
L U=-\left(\Delta^{2} u_{n-1}^{i}-\delta^{2} \nabla^{2} u_{n}^{i-1}\right)=\lambda u_{n}^{i}, \quad(i, n) \in[1, N] \times[1, m T],
$$

with the boundary conditions (11) and (12).

It is well known that the eigenvalue problem

$$
\left\{\begin{array}{l}
-\Delta^{2} x_{n-1}=\gamma x_{n}, \quad n \in[1, N] \\
x_{0}=x_{N+1}=0
\end{array}\right.
$$

has the eigenvalues

$$
\gamma_{k}=4 \sin ^{2} \frac{k \pi}{2(N+1)}, \quad k \in[1, N]
$$

and that the eigenvalue problem

$$
\left\{\begin{array}{l}
-\Delta^{2} x_{n-1}=\eta x_{n}, \quad n \in[1, m T] \\
x_{0}=x_{m T}, \quad x_{1}=x_{m T+1}
\end{array}\right.
$$

has the eigenvalues

$$
\eta_{l}=4 \sin ^{2} \frac{(l-1) \pi}{m T}, \quad l \in[1, m T] .
$$

See Cheng [24]. Thus, we see that all eigenvalues of the linear problem (16)-(11)-(12) are

$$
\lambda_{k l}=4 \sin ^{2} \frac{(l-1) \pi}{m T}-4 \delta^{2} \sin ^{2} \frac{k \pi}{2(N+1)}
$$

for $k \in[1, N]$ and $l \in[1, m T]$. That is,

$$
\sigma(L)=\left\{4\left[\sin ^{2} \frac{(l-1) \pi}{m T}-\delta^{2} \sin ^{2} \frac{k \pi}{2(N+1)}\right],(k, l) \in[1, N] \times[1, m T]\right\}
$$

We note that

$$
\begin{aligned}
& \gamma_{\max }=\max _{k \in[1, N]} \gamma_{k}=4 \sin ^{2} \frac{N \pi}{2(N+1)}, \\
& \gamma_{\min }=\min _{k \in[1, N]} \gamma_{k}=4 \sin ^{2} \frac{\pi}{2(N+1)}, \\
& \eta_{\max }=\max _{l \in[1, m T]} \eta_{l}= \begin{cases}4, & m T \text { is even, } \\
4 \cos ^{2} \frac{\pi}{m T}, & m T \text { is odd }\end{cases}
\end{aligned}
$$

and

$$
\eta_{\min }=\min _{l \in[1, m T]} \eta_{l}=0 .
$$


Thus, we have

$$
\begin{aligned}
\lambda_{\max } & =\max _{(k, l) \in[1, N] \times[1, m T]} \lambda_{k l} \\
& =\eta_{\max }-\delta^{2} \gamma_{\min } \\
& = \begin{cases}4\left(1-\delta^{2} \sin ^{2} \frac{\pi}{2(N+1)}\right), & m T \text { is even, } \\
4\left(\cos ^{2} \frac{\pi}{m T}-\delta^{2} \sin ^{2} \frac{\pi}{2(N+1)}\right), & m T \text { is odd, }\end{cases}
\end{aligned}
$$

and

$$
\lambda_{\min }=\min _{(k, l) \in[1, N] \times[1, m T]} \lambda_{k l}=-4 \delta^{2} \sin ^{2} \frac{N \pi}{2(N+1)} .
$$

In this paper, we require $\sigma(L) \cap(0, \infty) \neq \emptyset$ and $\sigma(L) \cap(-\infty, 0) \neq \emptyset$. Clearly, $\sigma(L) \cap(-\infty, 0) \neq$ $\emptyset$, thus, we only assume that the condition

$$
\begin{cases}\delta \sin \frac{\pi}{2(N+1)}<1, & m T \text { is even, } \\ \delta \sin \frac{\pi}{2(N+1)}<\cos \frac{\pi}{m T}, & m T \text { is odd, }\end{cases}
$$

holds, where $\delta>0$. On the other hand, we also need to suppose that the conditions

$$
\sin ^{2} \frac{(l-1) \pi}{m T} \neq \delta^{2} \sin ^{2} \frac{k \pi}{2(N+1)}
$$

hold for $(k, l) \in[1, N] \times[1, m T]$. In this case, we have $0 \notin \sigma(L)$. Throughout this paper, we always assume that the conditions (18) and (19) hold.

The abstract critical point theorem plays an important role in proving our main results. Let $E$ be a Hilbert space with norm $\|\cdot\|$ and inner product $\langle\cdot, \cdot\rangle$ and have an orthogonal decomposition $E=N \oplus N^{\perp}$, where $N \in E$ is a closed and separable subspace. Since $N$ is separable, we can define a new norm $|v|_{\omega}$ satisfying $|v|_{\omega} \leq\|v\|$ for all $v \in N$ and such that the topology induced by this norm is equivalent to the weak topology of $N$ on bounded subset of $N$. For $u=v+w \in E$ with $v \in N$ and $w \in N^{\perp}$, we define $|u|_{\omega}^{2}=|v|_{\omega}^{2}+\|w\|^{2}$, then $|u|_{\omega} \leq\|u\|$ for $u \in E$. Particularly, if $\left\{u_{n}=v_{n}+w_{n}\right\}_{n=1}^{\infty} \in E$ is $|\cdot|_{\omega}$-bounded and $u_{n} \rightarrow_{|\cdot|_{\omega}} u$, then $v_{n} \rightarrow v$ weakly in $N, w_{n} \rightarrow w$ strongly in $N^{\perp}, u_{n} \rightarrow v+w$ weakly in $E$ (see [21]).

Let $E=E^{-} \oplus E^{+}, z_{0} \in E^{+}$with $\left\|z_{0}\right\|=1$. For any $u \in E$, we write $u=u^{-} \oplus s z_{0} \oplus w^{+}$with $u^{-} \in E^{-}, s \in R, w^{+} \in\left(E^{-} \oplus R z_{0}\right)^{\perp}:=E_{1}^{+}$. For $R>0$, let

$$
Q=\left\{u=u^{-}+s z_{0} \mid s \in R^{+}, u^{-} \in E^{-},\|u\|<R\right\}
$$

with $p_{0}=s_{0} z_{0} \in Q, s_{0}>0$. We define

$$
D=\left\{u=s z_{0}+w^{+} \mid s \geq 0, w^{+} \in E_{1}^{+},\left\|s z_{0}+w^{+}\right\|=s_{0}\right\} .
$$

For $I \in C^{1}(E, R)$, define $h:[0,1] \times \bar{Q} \rightarrow E$ is $|\cdot|_{\omega}$-continuous, $h(0, u)=u, I(h(s, u)) \leq I(u)$ for $u \in \bar{Q}$, for any $\left(s_{0}, u_{0}\right) \in[0,1] \times \bar{Q}$, there is a $|\cdot|_{\omega}$-neighborhood $U_{\left(s_{0}, u_{0}\right)}$ such that

$$
\left\{u-h(t, u) \mid(t, u) \in U_{\left(s_{0}, u_{0}\right)} \cap[0,1] \times \bar{Q}\right\} \subset E_{\text {fin }},
$$


where $E_{\text {fin }}$ denotes various finite-dimensional subspaces of $E$ whose exact dimensions are irrelevant and depend on $\left(s_{0}, u_{0}\right)$. Denote

$$
\Gamma=\{h \mid h:[0,1] \times \bar{Q} \rightarrow E\},
$$

then $\Gamma \neq \emptyset$ since $i d \in \Gamma$.

The variant weak linking theorem is as follows.

Lemma 2 (see [21]) The family of $C^{1}$-functional $\left\{H_{\lambda}\right\}$ has the form

$$
H_{\lambda}(u)=I(u)-\lambda K(u) \quad \text { for } \lambda \in[1,2] .
$$

\section{Assume that}

(a) $K(u) \geq 0, u \in E, H_{1}=H$;

(b) $I(u) \rightarrow \infty$ or $K(u) \rightarrow \infty$ as $\|u\| \rightarrow \infty$;

(c) $H_{\lambda}$ is $|\cdot|_{\omega}$-upper semicontinuous, $H_{\lambda}^{\prime}$ is weakly sequentially continuous on $E$. Moreover, $H_{\lambda}$ maps bounded sets to bounded sets;

(d) $\sup _{\partial Q} H_{\lambda} \leq \inf _{D} H_{\lambda}$ for $\lambda \in[1,2]$.

Then, for almost all $\lambda \in[1,2]$, there exists a sequence $\left\{u_{n}\right\}$ such that

$$
\sup _{n}\left\|u_{n}\right\|<\infty, \quad H_{\lambda}^{\prime}\left(u_{n}\right) \rightarrow 0, \quad H_{\lambda}\left(u_{n}\right) \rightarrow c_{\lambda}
$$

where

$$
c_{\lambda}=\inf _{h \in \Gamma} \sup _{u \in Q} H_{\lambda}(h(1, u)) \in\left[\inf _{D} H_{\lambda}, \sup _{\bar{Q}} H\right] .
$$

\section{Main result}

First of all, we state the following conditions.

(i) $x f(n, x) \geq 0$ for $x \in R$ and $n \in Z$.

(ii) There exist $0<a<1$ and $r>0$ such that

$$
\int_{0}^{x} f(j, s) d s \leq \frac{a \sigma_{\min }^{+}}{2} x^{2} \quad \text { for }|x| \leq r .
$$

(iii) There exist $\rho>0$ and $d>1$ such that

$$
\int_{0}^{x} f(j, s) d s \geq \frac{d \sigma_{\max }^{+}}{2} x^{2} \quad \text { for }|x|>\rho
$$

where

$$
\sigma_{\min }^{+}=\min \left\{\lambda_{k l}>0,(k, l) \in[1, N] \times[1, m T]\right\}
$$

and

$$
\sigma_{\max }^{+}=\max \left\{\lambda_{k l}>0,(k, l) \in[1, N] \times[1, m T]\right\} .
$$


Theorem 1 The function $f: Z \times R \rightarrow R$ is continuous about the second variable and there exists a positive integer $T$ such that

$$
f(n+T, \cdot)=f(n, \cdot) \quad \text { for } n \in Z \text {. }
$$

Suppose that the above conditions (i)-(iii), (18), and (19) hold. Then for any positive integer $m$, for problem (10)-(12) there at least exists a non-zero time $m T$-periodic solution.

Proof In view of Lemma 2, we need to prove that the conditions (a)-(d) hold. First of all, we give some symbols.

When the conditions (18) and (19) hold, we can denote

$$
\begin{aligned}
& \sigma^{+}(L)=\left\{\lambda_{k l}>0,(k, l) \in[1, N] \times[1, m T]\right\}, \\
& \sigma^{-}(L)=\left\{\lambda_{k l}<0,(k, l) \in[1, N] \times[1, m T]\right\}, \\
& \sigma_{\min }^{+}=\min \left\{\lambda_{k l}>0,(k, l) \in[1, N] \times[1, m T]\right\}, \\
& \sigma_{\max }^{+}=\max \left\{\lambda_{k l}>0,(k, l) \in[1, N] \times[1, m T]\right\}, \\
& \sigma_{\min }^{-}=\min \left\{\lambda_{k l}<0,(k, l) \in[1, N] \times[1, m T]\right\},
\end{aligned}
$$

and

$$
\sigma_{\max }^{-}=\max \left\{\lambda_{k l}<0,(k, l) \in[1, N] \times[1, m T]\right\} .
$$

Clearly, we have

$$
\begin{aligned}
& \sigma_{\min }^{-}<\sigma_{\max }^{-}<0<\sigma_{\min }^{+}<\sigma_{\max }^{+}, \\
& \sigma(L)=\sigma^{+}(L) \cup \sigma^{-}(L),
\end{aligned}
$$

and

$$
\sigma^{+}(L) \cap \sigma^{-}(L)=\emptyset .
$$

That is, the linear operator $L=-\left(\Delta^{2}-\delta^{2} A\right)$ has a sequence of eigenvalues

$$
\begin{aligned}
\sigma_{\min }^{-} & =\lambda_{-p} \leq \lambda_{-p+1} \leq \cdots \leq \lambda_{-1}=\sigma_{\max }^{-} \\
& <0<\sigma_{\min }^{+}=\lambda_{1} \leq \lambda_{2} \leq \cdots \leq \lambda_{q}=\sigma_{\max }^{+}
\end{aligned}
$$

and the corresponding eigenvectors $\Upsilon_{j}$ for $j=-p, \ldots,-1,1, \ldots, q$. (Clearly, we have $p+q=$ $m T N$.)

Let

$$
E^{-}=\left\{\sum_{i=1}^{p} c_{i} \Upsilon_{-i} \mid c_{i} \in R\right\} \quad \text { and } \quad E^{+}=\left\{\sum_{i=1}^{q} c_{i} \Upsilon_{i} \mid c_{i} \in R\right\} .
$$

Then $E_{m T}=E=E^{-} \oplus E^{+}$and for any $U \in E_{m T}$ we have $U=U^{-}+U^{+}$, where $U^{-} \in E^{-}$and $U^{+} \in E^{+}$. Clearly, we have also $E^{0}=\operatorname{ker} L=\{0\}$. 
For any $U, V \in E_{m T}, U=U^{+}+U^{-}$and $V=V^{+}+V^{-}$, we can define an equivalent new inner product $\langle\cdot\rangle$ and the corresponding norm $\|\cdot\|$ in $E_{m T}$ by

$$
\langle U, V\rangle=\left\langle L U^{+}, V^{+}\right\rangle_{m T}-\left\langle L U^{-}, V^{-}\right\rangle_{m T} \quad \text { and } \quad\|U\|=\langle U, U\rangle^{1 / 2}
$$

see [25]. Therefore, $H$ can be rewritten as

$$
\begin{aligned}
H(U) & =\frac{1}{2}\left(\left\|U^{+}\right\|^{2}-\left\|U^{-}\right\|^{2}\right)-\Psi(U) \\
& =\frac{1}{2}\left\|U^{+}\right\|^{2}-\left(\frac{1}{2}\left\|U^{-}\right\|^{2}+\Psi(U)\right),
\end{aligned}
$$

where

$$
\Psi(U)=\sum_{j=1}^{m T} \sum_{i=1}^{N} \int_{0}^{u_{j}^{i}} f(j, s) d s=\sum_{j=1}^{m T} H\left(j, U_{j}\right)
$$

In order to apply Lemma 2, we consider the family of functional defined by

$$
H_{\lambda}(U)=\frac{1}{2}\left\|U^{+}\right\|^{2}-\lambda\left(\frac{1}{2}\left\|U^{-}\right\|^{2}+\Psi(U)\right) \quad \text { for } \lambda \in[1,2]
$$

In the following, we give the proofs for the conditions (a)-(d) in Lemma 2.

(a) $K(U) \geq 0, U \in E_{m T}, H_{1}=H$.

Let

$$
K(U)=\frac{1}{2}\left\|U^{-}\right\|^{2}+\Psi(U)
$$

When $x f(n, x) \geq 0$, we find that (a) holds.

(b) $I(U) \rightarrow \infty$ or $K(U) \rightarrow \infty$ as $\|U\| \rightarrow \infty$. We will prove that $K(U) \rightarrow \infty$ as $\|U\| \rightarrow \infty$. In fact, this is clear when the condition (iii) holds.

(c) $H_{\lambda}$ is $|\cdot|_{\omega}$-upper semicontinuous, $H_{\lambda}^{\prime}$ is weakly sequentially continuous on $E$. Moreover, $H_{\lambda}$ maps bounded sets to bounded sets.

The condition (iii) implies that

$$
\begin{aligned}
H_{\lambda}(U) & =\frac{1}{2}\left\|U^{+}\right\|^{2}-\lambda\left(\frac{1}{2}\left\|U^{-}\right\|^{2}+\Psi(U)\right) \\
& \leq \frac{1}{2}\left\|U^{+}\right\|^{2}-\frac{1}{2}\left\|U^{-}\right\|^{2}-\Psi(U) \\
& \leq \frac{\sigma_{\max }^{+}}{2}\|U\|_{m T}^{2}-\frac{d \sigma_{\max }^{+}}{2}\|U\|_{m T}^{2} \\
& =-(d-1) \frac{\sigma_{\max }^{+}}{2}\|U\|_{m T}^{2} \rightarrow-\infty \quad \text { as }\|U\|_{m T} \rightarrow \infty .
\end{aligned}
$$

Thus, if $U_{n} \rightarrow_{\mid \cdot \omega_{\omega}} U$ and $H_{\lambda}\left(U_{n}\right) \geq a$, then $H_{\lambda}(U) \geq a$, which means that $H_{\lambda}$ is $|\cdot|_{\omega}$-upper semicontinuous. The other cases are clear.

(d) $\sup _{\partial Q} H_{\lambda} \leq \inf _{D} H_{\lambda}$ for $\lambda \in[1,2]$. 
Note by the condition (ii), letting $s_{0}=r$, for $U \in S=\left\{U \mid U \in E^{+},\|U\|=s_{0}\right\}$ we have

$$
\begin{aligned}
H_{\lambda}(U) & =\frac{1}{2}\left\|U^{+}\right\|^{2}-\lambda \Psi(U) \\
& \geq \frac{\sigma_{\min }^{+}}{2}\|U\|_{m T}^{2}-2 \sum_{j=1}^{m T} \sum_{i=1}^{N} \int_{0}^{u_{j}^{i}} f(j, s) d s \\
& \geq \frac{\sigma_{\min }^{+}}{2}\|U\|_{m T}^{2}-\frac{a \sigma_{\min }^{+}}{2}\|U\|_{m T}^{2} \\
& =(1-a) \frac{\sigma_{\min }^{+}}{2}\|U\|_{m T}^{2} .
\end{aligned}
$$

Let $z_{0}=\Upsilon_{1} /\left\|\Upsilon_{1}\right\|$ and

$$
D=\left\{U \mid U=s z_{0}+W^{+}, s \geq 0, W^{+} \in E_{1}^{+} \text {and }\|U\|=s_{0}\right\}
$$

we have $D \subset S$, which implies that

$$
\inf _{D} H_{\lambda}(U)>0
$$

Now, we choose $\rho>0$ of the condition (ii) and let

$$
Q=\left\{U \mid U=U^{-}+s z_{0}, s \geq 0, U^{-} \in E^{-} \text {and }\|U\|<\rho\right\} .
$$

For $U \in \partial Q$, we have

$$
\begin{aligned}
H_{\lambda}(U) & =\frac{1}{2}\left\|U^{+}\right\|^{2}-\lambda\left(\frac{1}{2}\left\|U^{-}\right\|^{2}+\Psi(U)\right) \\
& \leq-(d-1) \frac{\sigma_{\max }^{+}}{2}\|U\|_{m T}^{2}<0 .
\end{aligned}
$$

That is, the condition (d) holds.

In view of Lemma 2, we find that for almost all $\lambda \in[1,2]$ there exists a sequence $\left\{U^{(n)}\right\} \subset$ $\bar{Q}$ such that

$$
\sup _{n}\left\|U^{(n)}\right\|<\infty, \quad H_{\lambda}^{\prime}\left(U^{(n)}\right) \rightarrow 0
$$

and

$$
H_{\lambda}\left(U^{(n)}\right) \rightarrow c_{\lambda} \in\left[\frac{1}{2}(1-a) r \sigma_{\min }^{+}, \sup _{\bar{Q}} H_{\lambda}(U)\right] .
$$

Note that the function $H_{\lambda}(U)$ is finite dimensional continuous, thus, there exist $\left\{U^{(n)}\right\} \subset \bar{Q}$ and $U^{*} \in \bar{Q}$ such that

$$
\lim _{n \rightarrow \infty} H_{1}\left(U^{(n)}\right)=\lim _{n \rightarrow \infty} H\left(U^{(n)}\right)=\lim _{n \rightarrow \infty} H\left(U^{*}\right)=c_{1}>0 .
$$

The proof is complete. 
Remark 1 The symbols $\sigma_{\min }^{-}$and $\sigma_{\max }^{-}$have not been used. In fact, similarly, if we discuss the functional $-H(U)$, then the corresponding result can also be obtained. It is omitted.

Remark 2 From the proofs of (a)-(d), we can see that the conditions (ii)-(iii) need only to be hold locally. For convenience, we use the present state.

Remark 3 Our result is new; see Deng et al. [6] and Yan et al. [7]. Clearly, the sublinear case can also be established. It will be omitted.

Remark 4 For an $m T$-dimensional discrete system of the form

$$
\Delta^{2} U_{n-1}+B U_{n}+\nabla V\left(n, U_{n}\right)=0, \quad n \in Z,
$$

when $B$ is a symmetric positive definite, negative definite, or infinite definite matrix, our method is also valid.

Remark 5 The superlinear condition (iii) cannot be used for the corresponding continuous wave equations or the continuous Hamiltonian systems because their eigenvalues are unbounded.

Remark 6 Our method is not suitable for the corresponding continuous wave equations. When $\omega^{2}$ is irrational, the eigenvalues of the operator $\partial_{t t}-\partial_{x x}$ accumulate to 0 . However, if $\omega^{2}$ is rational, the number of 0 -spectrum is infinite.

Remark 7 All conditions of Theorem 1 are easily satisfied. For the conditions (18) and (19), for example, let $\delta=1, m=1$ and $T=2$, the condition (18) clearly holds for any $N$. At the same time, note that

$$
\sin ^{2} \frac{(1-1) \pi}{2}=0, \quad \sin ^{2} \frac{(2-1) \pi}{2}=1
$$

and

$$
0<\sin ^{2} \frac{k \pi}{2(N+1)}<1
$$

for all $k \in[1, N]$. Thus, the condition (19) also holds. For the nonlinear term, the conditions (i)-(iii) are also easy satisfied. For example, for $\delta=1, m=1, T=2$, and $N=2$, we have

$$
\sigma(L)=\left\{-\frac{3}{4},-\frac{1}{4}, 1,3\right\}, \quad \sigma_{\min }^{+}=1, \quad \text { and } \quad \sigma_{\max }^{+}=3
$$

In this case, we let $f(-x)=-f(x)$ and

$$
f(x)= \begin{cases}\frac{x}{4}, & 0 \leq x<1 \\ \frac{59}{8} x-\frac{57}{8}, & 1 \leq x \leq 3 \\ 5 x, & x>3\end{cases}
$$

Then all conditions of Theorem 1 are satisfied. However, such a function is not valid for the corresponding continuous wave equations or the continuous Hamiltonian systems. 


\section{Competing interests}

The authors declare that they have no competing interests.

\section{Authors' contributions}

All authors contributed equally to the writing of this paper. All authors read and approved the final manuscript.

\section{Author details}

${ }^{1}$ School of Science, Tianjin University of Commerce, Tianjin, 300134, P.R. China. ${ }^{2}$ Department of Computing \& Information Systems, Department of Mathematics, Trent University, Peterborough, K9J 7B8, Canada. ${ }^{3}$ School of Computer Science \& Mathematics, Faculty of Science \& Engineering, University of Chester, Thornton Science Park, Pool Lane, Ince, Chester, CH2 $4 N U, U K$.

\section{Acknowledgements}

The authors would like to thank the anonymous referees for their careful review and helpful comments. This work was supported by the National Natural Science Foundation of China (No. 11371277). The project was completed while the first author was visiting Trent University and supported by a grant from the Natural Sciences and Engineering Research Council of Canada.

Received: 23 October 2014 Accepted: 6 April 2015 Published online: 17 April 2015

\section{References}

1. Vitt, VA: Distributed self-oscillating systems Zh. Tekh. Fiz. 4, 144-157 (1934) (in Russian)

2. Rabinowitz, P: Periodic solutions of nonlinear hyperbolic partial differential equations. Commun. Pure Appl. Math. 20, 145-205 (1967)

3. Brézis, H, Coron, JM: Periodic solutions of nonlinear wave equations and Hamiltonian systems. Am. J. Math. 103(3), 559-570 (1981)

4. Berti, $M$, Bolle, P: Cantor families of periodic solutions for completely resonant nonlinear wave equations. Duke Math. J. 134, 359-419 (2006)

5. Gentile, G, Mastropietro, V: Construction of periodic solutions of nonlinear wave equation with Dirichlet boundary conditions by the Lindstedt series method. J. Math. Pures Appl. 83, 1019-1065 (2004)

6. Deng, XQ, Shi, HP, Xie, XL: Periodic solutions of second order discrete Hamiltonian systems with potential indefinite in sign. Appl. Math. Comput. 218, 148-156 (2011)

7. Yan, $\mathrm{SH}, \mathrm{Wu}, \mathrm{XP}$, Tang, CL: Multiple periodic solutions for second-order discrete Hamiltonian systems. Appl. Math. Comput. 234, 142-149 (2014)

8. Jiang, LQ: Existence of periodic solutions for convex discrete Hamiltonian systems. Math. Sci. Res. J. 16(3), 64-81 (2012)

9. Guo, ZM, Yu, JS: Multiplicity results for periodic solutions to second-order difference equations. J. Dyn. Differ. Equ. 18(4), 943-960 (2006)

10. Poincaré, H: Les Méthodes Nouvelles de la Mécanique Céleste. Gauthier-Villars, Paris (1892)

11. Lyapunov, AM: Problème général de la stabilité du mouvement. Ann. Fac. Sci. Toulouse 2, 203-474 (1907)

12. Birkhoff, GD, Lewis, DC: On the periodic motions near a given periodic motion of a dynamical system. Ann. Math. 12, 117-133 (1933)

13. Moser, J: Periodic orbits near an equilibrium and a theorem by Alan Weinstein. Commun. Pure Appl. Math. 29, 727-747 (1976)

14. Weinstein, A: Normal modes $f$ or a nonlinear Hamiltonian system. Invent. Math. 20, 47-57 (1973)

15. Rabinowitz, P: Periodic solutions of Hamiltonian systems. Commun. Pure Appl. Math. 31, 157-184 (1978)

16. Ekeland, I, Lasry, JM: On the number of periodic trajectories for a Hamiltonian flow on a convex energy surface. Ann. Math. 112(2), 283-319 (1980)

17. Pugh, CC, Robinson, RC: The $C^{1}$ closing lemma, including Hamiltonians. Ergod. Theory Dyn. Syst. 3, $261-313$ (1983)

18. Biasco, L, Gregorio, LD: Periodic solutions of Birkhoff-Lewis type for the nonlinear wave equation. Discrete Contin. Dyn. Syst. suppl., 102-109 (2007)

19. Long, YM: Multiple solutions of perturbed superquadratic second order Hamiltonian systems. Trans. Am. Math. Soc. 311(2), 749-780 (1989)

20. Luan, S, Mao, A: Periodic solutions for a class of nonautonomous Hamiltonian systems. Nonlinear Anal. 61(8), 1413-1426 (2005)

21. Schechter, M, Zou, W: Weak linking theorems and Schrödinger equations with critical Sobolev exponent. ESAIM Control Optim. Calc. Var. 9, 601-619 (2003)

22. Chen, GW, Ma, SW: Periodic solutions for Hamiltonian systems without Ambrosetti-Rabinowitz condition and spectrum 0. J. Math. Anal. Appl. 379, 842-851 (2011)

23. Bai, L, Zhang, G: Nontrivial solutions for a nonlinear discrete elliptic equation with periodic boundary conditions. Appl. Math. Comput. 210, 321-333 (2009)

24. Cheng, SS: Partial Difference Equations. Taylor \& Francis, London (2003)

25. Stuart, CA: Bifurcation into spectral gaps. Bull. Belg. Math. Soc. suppl., pp. 59 (1995) 